\title{
Attenuation and Dispersion in Cancellous Bone: Implication in Developing Ultrasound-Guided Spinal Navigation
}

\section{Suoyuan Li}

Nanjing Medical University Affiliated Suzhou Hospital: Suzhou Municipal Hospital

\section{Peiyang Li}

Suzhou Institute of Biomedical Engineering and Technology

\section{Weiwei Shao}

Suzhou Institute of Biomedical Engineering and Technology

\section{Yang Jiao}

Suzhou Institute of Biomedical Engineering and Technology

\section{Zhiqiang Li}

Nanjing Medical University Affiliated Suzhou Hospital: Suzhou Municipal Hospital

\section{Wenjia Lu}

Suzhou Institute of Biomedical Engineering and Technology

\section{Yaoyao Cui}

Suzhou Institute of Biomedical Engineering and Technology

\section{Junjie Fan}

Suzhou Ninth People's Hospital

Jun Shen ( $D$ 18112603158@163.com )

Nanjing Medical University Affiliated Suzhou Hospital: Suzhou Municipal Hospital

\section{Research}

Keywords: Ultrasound, Spinal navigation, Cancellous bone

Posted Date: March 12th, 2021

DOI: https://doi.org/10.21203/rs.3.rs-282560/v1

License: (c) (1) This work is licensed under a Creative Commons Attribution 4.0 International License. Read Full License 


\section{Attenuation and dispersion in cancellous bone: implication}

\section{2 in developing ultrasound- guided spinal navigation}

3 Suoyuan $\mathrm{Li}^{1 \#}$, Peiyang $\mathrm{Li}^{2,3 \#}$, Weiwei $\mathrm{Shao}^{3}$, Yang $\mathrm{Jiao}^{3}$, Zhiqiang $\mathrm{Li}^{1}$, Wenjia $\mathrm{Lu}^{3}$,

$4 \quad$ Yaoyao Cui ${ }^{3}$, Junjie Fan $^{4}$, Jun Shen ${ }^{1 *}$

5 1. Department of Orthopedic Surgery, the Affiliated Suzhou Hospital of Nanjing

6 Medical University; Suzhou Municipal Hospital. Suzhou, Jiangsu, 215002, People’s

7 Republic of China.

8 2. Academy for Engineering \& Technology, Fudan University. Shanghai, China

9 3. Suzhou Institute of Biomedical Engineering and Technology (SIBET), Chinese

20 Academy of Science. Suzhou, Jiangsu, 215163, People's Republic of China.

4. Department of Orthopedic Surgery, Suzhou Ninth People's Hospital. Suzhou, Jiangsu, 215000, People's Republic of China.

\# These authors contributed equally to this work.

*Corresponding Author: Jun Shen, M.D, Ph.D.

Address: No.26, Daoqian Street, Department of Orthopedic Surgery, the Affiliated

Suzhou Hospital of Nanjing Medical University; Suzhou Municipal Hospital.

Suzhou, Jiangsu province, China, 215002.

Telephone: 008618112603158

E-mail: 18112603158@163.com 


\section{Abstract}

Background: The purpose of this study was to understand the acoustic properties of vertebral cancellous bone by combining micro-CT scan data and use this as the theoretical basis for ultrasonic navigation with posterior pedicle screw fixation during spinal fusion surgery.

Results: Two bovine spinal cancellous bone blocks and two human spinal allograft bone blocks simulated the condition of cancellous bone in the pedicle screw channel. Transmission experiments were performed on them, including amplitude, attenuation and sound velocity tests. Four unfocused wideband ultrasonic transducers were used for the detection, with the central frequencies of $2.2 \mathrm{MHz}, 2.5 \mathrm{MHz}, 3 \mathrm{MHz}$ and $12 \mathrm{MHz}$ respectively. The results were favorable and stable. The amplitude of the signal decreased with depth penetration $(\mathrm{p}<0.05)$. With the increase of frequency, sound attenuation and sound velocity increased $(\mathrm{p}<0.05)$.

Conclusions: In summary, the conclusions lay a theoretical foundation for the ultrasonic navigation system. However, how ultrasound navigation will facilitate pedicle screw insertion in spine surgery remains to be determined. Therefore, ultrasonic guided pedicle screw implantation is effective and promising in theory.

\section{Keywords}

Ultrasound; Spinal navigation; Cancellous bone

4 (1) 6 


\section{Background}

Pedicle screw fixation is a widely used posterior procedure for spinal stabilization in various situations, including spinal deformity, trauma, tumor and degenerative diseases (1). The misplacement rate of pedicle screws in the thoracic and lumbar vertebrae was reported to be $6.0-54.7 \%(2,3)$. Pedicle screw misplacement compromises neighboring structures such as nerves (and in some cases, vascular and spinal cord) (4), erodes long term biomechanical stability $(5,6)$, and causes adjacent spinal cord degeneration $(7,8)$.

We use a wide range of auxiliary surgical equipment to improve the accuracy of screw placement, such as C-arm X-ray machine, computer assisted surgery (CAS), neurophysiological monitoring. The advantage of X-ray fluoroscopy is that it can show the location of pedicle screws in real time, but exposure to radiation is detrimental to the surgeon's health. In addition, the two-dimensional images produced by X-ray perspective alone cannot provide sufficient spatial precision (9). CAS provides clear three-dimensional images and significantly reduces the screw displacement rate, but it is costly to use, takes up a large space, and requires complex registration and tracking methods, which have high requirements for surgeons (10). Finally, neurophysiological monitoring requires the tacit cooperation of surgical personnel, and the detection process is susceptible to external environment interference (11).

A desirable pedicle screw guidance tool may be free of ionizing radiation, economical, safe and easy to operate. Ultrasound transducers of different frequencies with the above characteristics have been attempted to develop into navigation approaches by several studies (12-16). Augmented reality surgical navigation with ultrasound-assisted registration for pedicle screw placement was also proposed (17). Recently, the combination of ultrasound and ultrasound and photoacoustic image 
guidance for pedicle screw placement has been proved feasible (18).To image the pedicle from the inside borehole, the ultrasound beam must penetrate through the cancellous bone to identify the boundary between the cancellous bone and outer context. Therefore, several studies have utilized through-transmission measurements to investigate the acoustic properties of trabecular bone inside pedicle using lowfrequency transducers $(0.5-3.5 \mathrm{MHz})$, the results of which showed ultrasonic attenuation increases with an increase in the bone volume or transducer frequency, and the trabecular and cortical bone can be detected in proper low frequency $(16,19)$. However, the limitations of ultrasound-guided pedicle screw include sound attenuation in the presence of cancellous bone, the presence of clutter and speckle noise, and poor signal-to-noise ratios (SNRs). Due to the difference of sound attenuation and sound velocity, the ability of traditional ultrasound imaging to detect deep-lying features under bone tissue is limited, so it needs to use multiple ultrasound slices as redundant information for registration (ie., 3D ultrasound imaging) $(18,20)$.

Cancellous bone is composed of bone trabeculae and bone marrow. The composition of the marrow varies with age, anatomical location, and disease state, demonstrating red marrow or yellow marrow $(21,22)$. Nicholson et al.(22) investigated the effect of marrow on quantitative ultrasound measurements using human calcaneal bone specimens, which indicated marrow significantly decreased ultrasound velocity, but increased attenuation and attenuation slope, especially to a greater extent at low BMD (Bone mineral density) values. In addition, marrow heterogeneity is another significant parameter affecting acoustic measurements. But in the intrapedicular imaging study by Aly et al. (19), the simulated measurements used water to replace the bone marrow, which cannot represent the marrow nature of composition variation and heterogeneity. 
Fung et al. (23) have proved that biological soft tissues are essentially viscoelastic materials, where they have both elastic solid and viscous fluid properties. To bone tissue, its solid properties mainly refer to various bone cells, bone fibers and bone trabeculae, while its fluid properties mainly refer to lipids in the bone cells or holes in the bone structure. These properties indicate that bone tissue also has viscoelastic properties, which are rarely considered in clinical examinations and can lead to errors. When stress is suddenly applied to the bone tissue, the bone tissue will deform. But due to the viscoelastic property, this deformation will continue to occur, known as creep. When the strain reaches a certain degree, the plastic deformation part will continue to increase, thus leading to the phenomenon of stress relaxation. The viscoelastic property of bone tissue has recently attracted much attention, and some studies have illustrated the effects of bone tissue viscoelasticity on ultrasound amplitude and sound velocity in cortical (24-26) and cancellous bone (27, 28).

The ultrasonic signal attenuates in the bone and is reflected by a large acoustic energy at the interface between soft tissue and bone tissue. The bone attenuation at the interface shadows features deeper in the tissue. In addition, using lower frequencies can result in considerable loss of image resolution. Therefore, a full understanding of the acoustic properties of cancellous bone is helpful to promote ultrasonic penetration of cancellous bone while maintaining image resolution.

The characteristics of biological tissues such as acoustic impedance, sound velocity, and acoustic attenuation have played a vital role in determining the usefulness of medical ultrasound detection and imaging technology. Quantitative ultrasound is portable, radiation-free, and a relatively inexpensive proxy for providing bone density; In addition, it can be used to assess the quality of bone properties (29). The wide application of this technique fully demonstrates the feasibility of ultrasonic transmission 
method to obtain cancellous bone (30). In the preparation of screw holes, the A-mode (Amplitude Mode) ultrasound images can be used to determine the location of the cancellous bone and cortical bone interface to display the upcoming early warning wave of perforation, to provide guidance to the surgeon. The CT scan parameters of cancellous bone can reflect the fine structure of cancellous bone density distribution, the trabecular bone trend, porosity, etc. Studying the correlation between the ultrasonic characteristics of cancellous bone and CT scan results can better promote the application of ultrasound in orthopedic navigation.

Therefore, the focus of this study was to better understand the acoustic characteristics of cancellous bone with different densities by using transmission measurements, and analyze the correlation between the ultrasonic characteristics of cancellous bone and the CT scan results, so as to determine the warning distance of pedicle screw channel.

\section{Results}

\section{Micro-CT scanning}

By micro-CT scanning of four different bone blocks, we obtained multiple parameters for two bovine and two human allograft bones (Fig. 1). The BMD of the two bovine bones had no significant difference and was higher than that of human allograft, while BMD of high density allograft was higher than that of low density allograft human bone (Table 1). BV/TV of low density allograft human bone $(26.11 \pm 0.03)$ was higher than that of low density human allograft bone $(14.21 \pm 0.02)$. Tb. Th and Th. $\mathrm{N}$ of human allograft bone (high density) were higher than human allograft bone (low density). Th. Sp and SMI of human allograft bone (high density) were lower than these of human allograft bone (low density). Nonetheless, there was no significant difference in the above indexes. However, the above parameters were not significantly different in 
the two bovine bones.

Table 1 The characteristics of each bone block of micro-CT scanning. (Mean \pm SD)

\begin{tabular}{|c|c|c|c|c|}
\hline & $\begin{array}{l}\text { Bovine } \\
\text { bone } 1\end{array}$ & $\begin{array}{l}\text { Bovine } \\
\text { bone } 2\end{array}$ & $\begin{array}{l}\text { Iuman allograft } \\
\text { bone (high } \\
\text { density) }\end{array}$ & $\begin{array}{l}\text { Human allograft } \\
\text { bone (low } \\
\text { density) }\end{array}$ \\
\hline BMD & $0.55 \pm 0.01$ & $0.54 \pm 0.01$ & $0.50 \pm 0.02$ & $0.48 \pm 0.01$ \\
\hline $\mathrm{BV} / \mathrm{TV}$ & $27.83 \pm 0.05$ & $21.34 \pm 0.03$ & $26.11 \pm 0.03$ & $14.21 \pm 0.02$ \\
\hline SMI & $1.08 \pm 0.01$ & $2.06 \pm 0.01$ & $1.05 \pm 0.01$ & $1.67 \pm 0.01$ \\
\hline Tb.Th & $0.31 \pm 0.01$ & $0.42 \pm 0.01$ & $0.22 \pm 0.00$ & $0.21 \pm 0.00$ \\
\hline Th.N & $0.90 \pm 0.00$ & $0.51 \pm 0.01$ & $1.19 \pm 0.01$ & $0.69 \pm 0.00$ \\
\hline Th.Sp & $0.90 \pm 0.01$ & $1.20 \pm 0.03$ & $0.60 \pm 0.00$ & $0.88 \pm 0.00$ \\
\hline
\end{tabular}

\section{Penetration amplitude and thickness of bone samples}

In Fig. 2, through ultrasonic transmission experiments on each bone sample, we found that in cancellous bone samples, the greater thickness, the greater attenuation of ultrasonic signal after penetration, and the lower the amplitude after penetration. Due to the anisotropy of trabeculae in cancellous bone, the attenuation of ultrasonic signal is relatively great when the thickness of bone sample is relatively large. As the thickness of cancellous bone sample decreases, the degree of trabecular anisotropy in the bone sample decreases, and the penetration amplitude of ultrasonic signal increases sharply. This phenomenon is particularly evident in relatively low-density bone samples.

\section{Acoustic attenuation and thickness of bone samples}

In this experiment, the sound attenuation coefficient increased with the thickness of each bone sample (Fig. 3). The reason is that the trabeculae in cancellous bone are anisotropic. When the thickness of bone sample is relatively large, the attenuation of ultrasonic signal is relatively large, and the acoustic attenuation coefficient measured by experiment is relatively large. As the thickness of cancellous bone sample decreased, 
the degree of trabecular anisotropy in the bone sample decreased, the penetration amplitude of ultrasonic signal increased sharply, and the sound attenuation coefficient measured by experiment was relatively small. This is especially true for relatively lower BMD bone samples. This is precisely caused by the relatively small trabecular anisotropy of the lower BMD samples.

Under the same thickness condition, the BMD of bovine bone was higher than that of allogeneic bone, and the acoustic attenuation coefficient of allogeneic bone obtained by the experiment was relatively smaller. For ultrasonic signals with different central frequencies and bone samples of different allografts, the differences in BMD were high, and the acoustic attenuation coefficient of the denser allografts was relatively high. For example, at $3 \mathrm{MHz}$ frequency, the acoustic attenuation of $2 \mathrm{~mm}$ high bone density allogeneic bone was $5.55 \pm 0.61$, which was much higher than the acoustic attenuation of $2 \mathrm{~mm}$ low bone density allogeneic bone $1.7 \pm 0.97(\mathrm{p}<0.05)$.

\section{Acoustic attenuation coefficient and ultrasonic frequency}

In the ultrasonic transmission experiment of bovine and allograft bones (Fig. 4), it was found that in the same bone block of the same thickness, the sound attenuation coefficients of low-frequency ultrasound $(2.2 \mathrm{MHz}, 2.5 \mathrm{MHz}, 3 \mathrm{MHz})$ were significantly lower than those of high-frequency ultrasound $(12 \mathrm{MHz})(\mathrm{p}<0.05)$. We believe that the ultrasound signal enters the cancellous bone pores, being absorbed and refracted. The wavelength decreased as the ultrasonic frequency increased, and the attenuation increased sharply when the wavelength approached the cancellous bone pore size.

In bovine and allograft bone, the sound attenuation of the thicker bone at the same frequency was greater.

\section{Ultrasonic frequency and ultrasonic velocity}

As shown in Fig. 5, the propagation wave speed $1400-1600 \mathrm{~m} / \mathrm{s}$ of cancellous 
bone is much slower than the propagation speed $3400-4200 \mathrm{~m} / \mathrm{s}$ of cortical bone, but it is close to the propagation speed of $1450 \mathrm{~m} / \mathrm{s}$ in bone marrow (31). This can be explained by the fact that the cancellous bone is not solid but has a spongy structure. With the increase of frequency, the average sound velocity in each bone sample increased significantly in the experiment $(\mathrm{p}<0.05)$. This phenomenon indicates that the propagation speed of ultrasound in cancellous bone is related to the center frequency of ultrasound.

At the frequencies of $2.2 \mathrm{MHz}, 2.5 \mathrm{MHz}, 3 \mathrm{MHz}$ and $12 \mathrm{MHz}$, there was statistically difference in ultrasonic sound velocity between the two bovine cancellous samples $(\mathrm{p}<0.05)$

However, in allograft bones, the sound speed of bone sample with low BMD was significantly higher than that of bone mass with high $\mathrm{BMD}$ at $2.2 \mathrm{MHz}, 2.5 \mathrm{MHz}, 3 \mathrm{MHz}$ and $12 \mathrm{MHz}(\mathrm{p}<0.05)$. We believe that the pore space of high BMD allograft bone is smaller than that of low BMD allograft bone, and the sound velocity is lower when ultrasound penetrates.

\section{Thickness of bone samples and ultrasonic velocity}

The correlation between the thickness of bone samples and ultrasonic velocity is shown in Fig. 6. In the same human allograft cancellous bone, the sound velocity did not change significantly with the increasing of thickness at the same ultrasonic frequency ( $p>0.05)$. However, in the same bovine cancellous bone, the sound velocity increased with the increasing of bone thickness in the same ultrasonic frequency. In transmission experiments, ultrasonic signals at each frequency could penetrate human allograft bone blocks of up to $5.00 \mathrm{~mm}$, and bovine bone blocks of up to $8.00 \mathrm{~mm}$ at central frequencies of $2.2 \mathrm{MHz}$ and $2.5 \mathrm{MHz}$. With the increasing of bone mass thickness, the closer the edge of cancellous bone mass is to cortical bone, the 
higher the bone density is and the higher the porosity is, so the sound velocity is relatively faster.

\section{Multiple regression analysis}

Bone tissue is an anisotropic and heterogeneous fluid porous composite medium with complex structure and special acoustic properties. The propagation attenuation of ultrasound in bone tissue is large and penetrability is poor, and we found that there is a serious dispersion phenomenon in the propagation process: low frequency ultrasonic attenuation is small; high frequency ultrasonic attenuation is large. As a viscoelastic medium, severe dispersion attenuation and distortion of bone tissue are the key technical problems in the evaluation and imaging of cancellous bone by ultrasonic transmission experiments. In order to accurately measure the values of nail canal in pedicle ultrasonic navigation, the sound velocity correction factor of ultrasonic cancellous bone propagation was introduced for the first time to correct the errors caused by the difference in viscoelasticity of cancellous bone. Multiple regression analysis was used to quantitatively study the correlation between acoustic characteristics of cancellous bone and CT scanning characteristics. The acoustic characteristics include amplitude (Am), attenuation (At), sound velocity ( $\left.{ }^{c_{s}}\right)$ and frequency (f), and the bone characteristics of CT-scan include BMD ( $\left.\rho_{s}\right), \mathrm{BV} / \mathrm{TV}(\mathrm{B})$, SMI (S), Tb.Th, Th.N, Th.Sp (T) and thickness (D). Through statistical calculation, we found that these data were in line with the linear regression model, and the following regression equations were fitted:

$$
A \mathrm{~m}=336.65-518.08 \rho_{s}-3.89 f-6.58 D
$$

Equation (1) illustrated that amplitude (Am) and BMD ( $\left.\rho_{s}\right)$, frequency (f), thickness (D) were negatively correlated. 
Equation (2) illustrated that attenuation (At) and frequency (f), thickness (D), BV/TV (B), SMI (S) were negatively correlated.

$$
c_{S}=1131.56+35.54 f+186.48 T
$$
positively correlated.

\section{Discussion}

Bone tissue is an anisotropic and heterogeneous fluid porous composite medium with complex structure and special acoustic properties. The propagation attenuation of ultrasound in bone tissue is large and penetrability is poor, and we found that there is a serious dispersion phenomenon in the propagation process: low frequency ultrasonic attenuation is small; high frequency ultrasonic attenuation is large. As a viscoelastic medium, severe dispersion attenuation and distortion of bone tissue are the key technical problems in the evaluation and imaging of cancellous bone by ultrasonic transmission experiments. In order to accurately measure the values of nail canal in pedicle ultrasonic navigation, the sound velocity correction factor of ultrasonic frequency alternate strain, but the strain of the sinusoidal stress imposed by the phase lag behind, called lag phenomenon. Different frequency of the sinusoidal stress can lead to different lag effect, ultimately reflected as dispersion. In traditional studies, the influence of the dispersion effect of bone tissue is often neglected, but in some precision clinical surgeries, such as pedicle screw implantation, the imaging results of bone tissue 
are required to be more accurate. Therefore, the deviation of ultrasonic velocity caused by dispersion effect must be considered. In our paper, it is found that the bovine bone ultrasonic sound velocity corresponding to $2.2 \mathrm{MHz}, 2.5 \mathrm{MHz}, 3 \mathrm{MHz}$ and $12 \mathrm{MHz}$ is $1388.34 \mathrm{~m} / \mathrm{s}, 1419.60 \mathrm{~m} / \mathrm{s}, 1467.50 \mathrm{~m} / \mathrm{s}$ and $1708.81 \mathrm{~m} / \mathrm{s}$. The speed of sound is increased with frequency. These results reveal that during pedicle ultrasound imaging, it is necessary to consider the influence of dispersion effect brought by bone viscoelasticity, and finally provide a frequency- based correction factor base on frequency for ultrasonic imaging results.

At the time of pedicle screw implantation, a complete wall of the screw canal is surrounded by cancellous bone. As the diameter of the screw hole expands, the closer it is to the bone cortex, the bone density of cancellous bone also increases. The bone density of the human vertebral cortex was $1.59 \pm 0.18 \mathrm{~g} / \mathrm{cm}^{3}$. We selected fresh bovine cancellous bone whose bone density was between the human cortical bone density and the cancellous bone density of the human vertebral body, and simulated the bone condition of the junction between the cancellous bone and the cortical bone in the pedicle screw canal during the operation. In the selection of human spinal cancellous allograft bone, the commercial allograft bone has been fully washed and the organic component in the trabecular space has been greatly reduced, which is very similar to the case of the cancellous bone in the screw canal wall during the operation. Among them, the cancellous bone mass with higher density simulated the cancellous bone condition of the nail wall in patients with normal bone density, while the bone mass with lower bone density simulated the cancellous bone condition of pedicle screw in patients with osteoporosis. Through ingenious design, we have replaced the complex structure of pedicle screw canal in actual surgery with regular bone blocks of different properties, making its ultrasonic characteristics easier to measure and further laying a foundation 
for the development of ultrasonic spinal navigation technology.

Micro-CT can obtain the physical parameters of cancellous bone mass. BV/TV is a common index for evaluating cancellous bones. For cancellous bone, this ratio can reflect the amount of trabecular bone mass in different samples. An increase in this value indicates that bone anabolism is greater than catabolism and bone mass increases, and vice versa, thus indirectly reflecting bone metabolism. In human allogeneic bone with low bone density, the BV/TV value of low-density bone mass is significantly lower than that of high-density bone, and has higher ultrasonic amplitude and lower acoustic attenuation at the same thickness and frequency. This further indicates that ultrasound is more likely to transmit low bone mass cancellous bone. Th. $\mathrm{N}, \mathrm{Tb}$. Th and Th. Sp can be calculated from the microstructure of trabeculae, which are the main indexes to evaluate the spatial morphological structure of trabeculae. When osteoporosis occurs, Th. N and Tb. Th values decrease and Th. Sp increase. SMI is a parameter to describe the proportion of plate structure and rod structure in trabecular structure. If the trabecular structure is mainly lamellar structure, then SMI is close to 0. On the other hand, SMI is closer to 3 if it is predominantly rod-shaped trabeculae. In osteoporosis, the trabecular bone changes from plate to rod, and the value increases.

For bone samples, the higher the bone density, the lower the penetration amplitude. The bone density of cattle was higher than that of allogeneic bone, and the penetration amplitude of cattle bone was lower than that of allogeneic bone. There was no significant difference in the density of bovine bone samples, so the amplitude after penetration was basically similar. In allograft bone, the penetration amplitude of samples with high BMD was lower than that of samples with low BMD. These results suggest that the denser the microstructure of cancellous bone, the less penetrable the ultrasound is. 
The amplitudes of each cancellous bone mass were significantly decreased under

the $12 \mathrm{MHz}$ frequency probe, and the amplitudes of each bone mass were slightly different. This reflects the sound attenuation of high-frequency ultrasound in the process of penetration of cancellous bone mass. We consider that the wavelength of high-frequency ultrasound is close to the pore size of cancellous bone, which is easily absorbed by cancellous bone, resulting in the sharp attenuation of sound waves.

\section{Conclusions}

In our study, it was found that the thicker cancellous bone block, the higher bone density and the higher ultrasonic frequency, the greater attenuation of ultrasonic signal and the lower amplitude after penetration. In addition, our study further explored the influence law of viscoelastic information in bone tissue on the ultrasonic dispersion effect. Higher viscoelasticity would bring higher dispersion effect, which would lead to great changes in ultrasonic sound velocity at different frequencies. In the same bone sample, the speed of sound increased with the increase of ultrasonic frequency. However, further research is needed to make ultrasound navigation an efficient, portable, real-time imaging system that can accurately guide pedicle screw implantation in spinal surgery. At present, ultrasound-guided pedicle screw implantation is theoretically effective and promising.

\section{Methods}

\section{Bone specimens}

To simulate the actual situation of pedicle screw holes, we selected two bovine vertebral cancellous bones and two human allograft cancellous bones. Two bovine vertebral cancellous bone masses were used to simulate the pedicle screw holes close to cortical bone in patients with normal bone density. A low bone density human allograft 
cancellous bone mass was used to simulate the innermost layers of pedicle holes in osteoporosis patients; and a normal bone density human allograft cancellous bone mass was used to simulate pedicle screw holes in patients with normal bone density. Fresh bovine bones were purchased from the animal experiment center of Nanjing Medical University. Human allograft bones were purchased from Shanxi ruiao biological materials co., LTD (registration certificate number: 20163460428). Cortical bone was removed and the thickness of reserved cancellous bone was all $8.00 \mathrm{~mm}$. The bone blocks were repeatedly soaked and rinsed with normal saline, and remained in degassed water for more than an hour to remove the air bubbles that may exist in the cancellous bone samples. In the process of ultrasonic transmission test, in order to ensure the consistency of the tested bone blocks and the accuracy of bone block thickness, two bovine cancellous bone blocks were successively thinned from $8.00 \mathrm{~mm}$ to $7.00 \mathrm{~mm}$, $5.00 \mathrm{~mm}$ and $2.00 \mathrm{~mm}$; two human allograft cancellous bone blocks were thinned successively from $8.00 \mathrm{~mm}$ to $6.50 \mathrm{~mm}, 5.00 \mathrm{~mm}, 3.00 \mathrm{~mm}$ and $2.00 \mathrm{~mm}$.

\section{Micro-CT scanning}

Micro-CT scanning (Skyscan) was performed on human allograft bone and bovine bone samples to obtain $\mathrm{CT}$ parameters of each bone sample. These parameters included BMD, SMI (Structural model index), Tb. Th (Trabecular thickness), Th. N (Trabecular number), Th. Sp (Trabecular separation), and BV/TV (Bone volume/tissue volume).

\section{Experimental system and procedures}

A custom-designed ultrasonic immersion system for through transmission measurements was applied in the current investigation and is shown in Fig. 7(A). The water tank was filled with deionized-degassed water maintained by a canister filter (Liqui-Cel ${ }^{\circledR}$ membrane 2.5x 8X-50 Fiber) and a custom-designed integrated filtration 
system and all experiments were carried out in degassed water at room temperature around $25^{\circ} \mathrm{C}$, and the speed of sound is $1497 \mathrm{~m} / \mathrm{s}$. The air bubbles in the water will scatter the ultrasonic waves; it will greatly affect the accuracy and reliability of the experimental results. Therefore, before the experiment, the pulse-echo measurements of the water medium was carried out to confirm that there was no millimeter-sized air bubbles into the water tank through the filtration system.

The cancellous bone chips were fixed on a four-dimensional control platform, and could perform $\mathrm{X}, \mathrm{Y}$, and $\mathrm{Z}$ translations, and rotation about the $\mathrm{Z}$-axis along with the platform. As indicated in Fig. 7(B), the transmitting transducer (custom-designed) and receiving Needle Hydrophone (SN2571, Precision Acoustics Ltd. UK) are placed coaxially on both sides of the bone fragment. The Needle Hydrophone is assembled on a customized fixture which can provide sufficient stability during the movement and attached to a micrometer. The transmitting transducer is fixed on the control platform which can perform $\mathrm{X}$ translation, $\mathrm{X}-\mathrm{Y}$ plane pitching adjusting, and rotation movement around the $\mathrm{Z}$ axis, and the control platform attached to a micrometer with the translation accuracy and rotation Angle accuracy $0.1 \mathrm{~mm}$ and $0.5^{\circ}$ respectively. Thus, the distance between the transducer, hydrophone and bone samples can be controlled by adjusting the consoles. Through the experimental setup, multiple locations of interest can be measured on the same cancellous bone sample, thereby reducing the experimental measurement error. The selected measurement positions were kept within the specimen boundaries to ensure that the main lobe of the ultrasound wave only encompassed cancellous bone.

The ultrasound data acquisition system comprised a ultrasonic pulser-receiver (Model CTS-8077PR, Guangdong Goworld Co., Ltd. CHINA), a wide bandwidth receiver Needle Hydrophone (Model NH0500, PA, Ltd., UK), a oscilloscope (Model 
DPO 5034, Tektronix, USA), and a personal computer (Fig. 7(A)). The ultrasonic transmission experiments were conducted on each bone sample with custom-designed ultrasonic probes(Suzhou GuoKe Ultra Medical Technology Co., Ltd, China): $2.2 \mathrm{MHz}$ (final dimension $3.0 \mathrm{~mm} \times 1.3 \mathrm{~mm} \times 2.0 \mathrm{~mm},-6 \mathrm{~dB}$ bandwidth of $44.36 \%$.), $2.5 \mathrm{MHz}$ (final dimension $5.0 \mathrm{~mm} \times 2.0 \mathrm{~mm} \times 2.5 \mathrm{~mm},-6 \mathrm{~dB}$ bandwidth of $50.08 \%$.), $3 \mathrm{MHz}$ (final dimension $2.0 \mathrm{~mm} \times 0.9 \mathrm{~mm} \times 1.6 \mathrm{~mm},-6 \mathrm{~dB}$ bandwidth of $48.26 \%$.) and $12 \mathrm{MHz}$ (final dimension $3.0 \mathrm{~mm} \times 1.0 \mathrm{~mm} \times 0.8 \mathrm{~mm},-6 \mathrm{~dB}$ bandwidth of $52.67 \%$.) respectively. The transducer was driven by the pulser/ receiver, and the radio frequency (RF) signal penetrating the bone sample was received by the wide band width receiver Needle Hydrophone and displayed on the oscilloscope. The RF data was saved as a .csv file for subsequent data processing.

The standard substitution technique was used to measure the ultrasonic characteristics of cancellous bone. As Fig. 8 shows, the principle is that when a cancellous bone sample of thickness $\mathrm{D}$ is inserted into the sound path between the transmitting and receiving transducers $\mathrm{T} 1$ and $\mathrm{T} 2$ submerged in water, the amplitude of the acoustic pulse received by $\mathrm{T} 2$ and its position in the time domain are relatively changed after the sample insertion. The sound velocity of the cancellous bone sample and water are $c_{s}$ and $c_{w}(1497 \mathrm{~m} / \mathrm{s})$, respectively, and the time shift of the received pulse caused by the insertion of the sample is $\Delta t$, then the sound velocity of the tissue sample is:

$$
c_{S}=\frac{D c_{w}}{D-\Delta t c_{w}}
$$

The sound velocity $\left({ }^{c_{s}}\right)$, acoustic impedance $\left({ }^{Z_{s}}\right)$, acoustic reflection coefficient $\left({ }^{r_{s}}\right)$ and acoustic attenuation $\left({ }^{\alpha(f)}\right)$ are important parameters that characterize the acoustic properties of biological tissue. The acoustic impedance $\left(Z_{s}\right)$ and acoustic 
reflection coefficient $\left({ }^{r_{s}}\right)$ were calculated using:

$$
\begin{aligned}
& Z_{s}=c_{s} \rho_{s} \\
& r_{s}==\frac{Z_{s}-Z_{w}}{Z_{s}+Z_{w}}
\end{aligned}
$$

Where, ${ }^{\rho_{s}}$ represented the density of bone sample, ${ }^{Z_{w}}$ represented the acoustic impedance of water. The attenuation was calculated using:

$$
\alpha(f)=20 \log _{10} \frac{Y_{\text {sig }}(f)}{Y_{\text {ref }}(f)} / D
$$

Where, ${ }^{Y_{s i}}(f)$ and ${ }^{Y_{r f}}(f)$ are the amplitude spectrums (magnitude of the Fourier transform) of the reference and bone signal, respectively, and D is the sample thickness.

\section{Transmission measurements}

When the ultrasonic wave is vertically incident to a large enough smooth flat interface, a reflected wave in the first medium will be generated in the opposite direction of the incident wave, and a transmitted wave in the second medium will be generated in the same direction as the incident wave.

The distances between the sample and transducers and the radius of the transducers are very important information. The size of the bone sample used in the experiment was greater than $20 \mathrm{~mm}$, while the maximum size of the ultrasonic transducer used in the experiment was less than $5 \mathrm{~mm}$. In order to ensure the accuracy and reliability of the experimental test data, the pulse transmission method is adopted in the experiment. In addition, the transducer should be as close to the bone sample as possible while the echo signal and the excitation pulse can be distinguished in the data analysis (Fig. 9).

The standard substitution technique was used to measure the ultrasonic characteristics of cancellous bone. The characteristics of cancellous bone at different 
ultrasonic frequencies were obtained by calculating the time and amplitude changes between the reference signal and the transmitted signal (Fig. 10).

\section{Statistical analysis}

All data are presented as mean \pm standard deviation (SD) and were analyzed using SPSS 9.1 software. Differences between each group were compared with a one-way ANOVA followed by a least significant difference (LSD) test with homogeneity of variance, or Dunnett T3 with heterogeneity of variance for multiple comparisons. Multiple regression analysis was used to quantitatively study the correlation between acoustic characteristics of cancellous bone and CT scanning characteristics. A p-value< 0.05 was regarded as statistically significant.

\section{References}

1. Boos N, Webb JK. Pedicle screw fixation in spinal disorders: a European view. Eur Spine J. $1997 ; 6(1): 2-18$.

2. Floccari LV, Larson AN, Crawford CH, 3rd, Ledonio CG, Polly DW, Carreon LY, et al. Which Malpositioned Pedicle Screws Should Be Revised? J Pediatr Orthop. 2018;38(2):110-5. 3. Li G, Lv G, Passias P, Kozanek M, Metkar US, Liu Z, et al. Complications associated with thoracic pedicle screws in spinal deformity. Eur Spine J. 2010;19(9):1576-84.

4. Li HM, Zhang RJ, Shen CL. Accuracy of Pedicle Screw Placement and Clinical Outcomes of Robot-assisted Technique Versus Conventional Freehand Technique in Spine Surgery From Nine Randomized Controlled Trials: A Meta-analysis. Spine (Phila Pa 1976). 2020;45(2):E111-E9. 5. Hecht N, Yassin H, Czabanka M, Föhre B, Arden K, Liebig T, et al. Intraoperative Computed Tomography Versus 3D C-Arm Imaging for Navigated Spinal Instrumentation. Spine. $2018 ; 43(5): 370-7$

6. Açikbaş SC, Arslan FY, Tuncer MR. The effect of transpedicular screw misplacement on late spinal stability. Acta neurochirurgica. 2003;145(11):949-54; discussion 54-5.

7. Park P, Garton HJ, Gala VC, Hoff JT, McGillicuddy JE. Adjacent segment disease after lumbar or lumbosacral fusion: review of the literature. Spine. 2004;29(17):1938-44. 
8. Aota Y, Kumano K, Hirabayashi S. Postfusion instability at the adjacent segments after rigid pedicle screw fixation for degenerative lumbar spinal disorders. Journal of spinal disorders. 1995;8(6):464-73.

9. Esfandiari H, Newell R, Anglin C, Street J, Hodgson AJ. A deep learning framework for segmentation and pose estimation of pedicle screw implants based on C-arm fluoroscopy. Int $\mathrm{J}$ Comput Assist Radiol Surg. 2018;13(8):1269-82.

10. Hernandez D, Garimella R, Eltorai AEM, Daniels AH. Computer-assisted Orthopaedic Surgery. Orthop Surg. 2017;9(2):152-8.

11. Mavrogenis AF, Papagelopoulos PJ, Korres DS, Papadopoulos K, Sakas DE, Pneumaticos S. Accuracy of pedicle screw placement using intraoperative neurophysiological monitoring and computed tomography. J Long Term Eff Med Implants. 2009;19(1):41-8.

12. Mujagić M, Ginsberg H, Cobbold R. Development of a method for ultrasound-guided placement of pedicle screws. IEEE transactions on ultrasonics, ferroelectrics, and frequency control. 2008;55(6):1267-76.

13. Kantelhardt S, Bock C, Larsen J, Bockermann V, Schillinger W, Rohde V, et al. Intraosseous ultrasound in the placement of pedicle screws in the lumbar spine. Spine. 2009;34(4):400-7.

14. Raphael D, Chang J, Zhang Y, Kudija D, Chen T, Shung K. A-Mode ultrasound guidance for pedicle screw advancement in ovine vertebral bodies. The spine journal : official journal of the North American Spine Society. 2010;10(5):422-32.

15. Ungi T, Moult E, Schwab J, Fichtinger G. Tracked ultrasound snapshots in percutaneous pedicle screw placement navigation: a feasibility study. Clinical orthopaedics and related research. 2013;471(12):4047-55.

16. Chen Z, Wu B, Zhai X, Bai Y, Zhu X, Luo B, et al. Basic study for ultrasound-based navigation for pedicle screw insertion using transmission and backscattered methods. PloS one. 2015;10(4):e0122392.

17. Ma L, Zhao Z, Chen F, Zhang B, Fu L, Liao H. Augmented reality surgical navigation with ultrasound-assisted registration for pedicle screw placement: a pilot study. International journal of computer assisted radiology and surgery. 2017;12(12):2205-15.

18. Gonzalez E, Jain A, Lediju Bell M. Combined ultrasound and photoacoustic image guidance 
of spinal pedicle cannulation demonstrated with intact ex vivo specimens. IEEE transactions on bio-medical engineering. 2020.

19. Aly AH, Ginsberg HJ, Cobbold RS. On ultrasound imaging for guided screw insertion in spinal fusion surgery. Ultrasound in medicine \& biology. 2011;37(4):651-64.

20. Wein W, Röper B, Navab N. Integrating diagnostic B-mode ultrasonography into CT-based radiation treatment planning. IEEE transactions on medical imaging. 2007;26(6):866-79.

21. Schnitzler CM, Mesquita J. Bone marrow composition and bone microarchitecture and turnover in blacks and whites. Journal of bone and mineral research : the official journal of the American Society for Bone and Mineral Research. 1998;13(8):1300-7.

22. Nicholson PH, Bouxsein ML. Bone marrow influences quantitative ultrasound measurements in human cancellous bone. Ultrasound in medicine \& biology. 2002;28(3):369-75.

23. Fung YC, Cowin SC. Biomechanics: Mechanical Properties of Living Tissues, 2nd ed. Journal of Applied Mechanics. 1994;61(4):1007-.

24. Haiat G, Grimal Q, Talmant M, Desceliers C, Soize C, Naili S, editors. Time-domain model of the ultrasonic wave propagation in an inhomogeneous anisotropic fluid/solid multilayer medium: application to cortical bone. Ultrasonics Symposium; 2010.

25. Bernard S, Grimal Q, Laugier P, editors. Measuring viscoelastic properties of cortical bone with resonant ultrasound spectroscopy. IEEE International Ultrasonics Symposium; 2012.

26. Neamtu A, Gozman-Pop IC, Vladaia D, Simoiu D, Bereteu L, editors. Determination of Bone Tissue Viscoelastic Properties by Vibroacoustic Measurements. 2020 IEEE 18th International Symposium on Intelligent Systems and Informatics (SISY); 2020.

27. Aygün H, Attenborough K, Postema M, Lauriks W, Langton CM. Predictions of angle dependent tortuosity and elasticity effects on sound propagation in cancellous bone. The Journal of the Acoustical Society of America. 2009;126(6):3286-90.

28. Wear KA. Mechanisms of Interaction of Ultrasound With Cancellous Bone: A Review. IEEE transactions on ultrasonics, ferroelectrics, and frequency control. 2020;67(3):454-82.

29. Glüer CC. Quantitative ultrasound techniques for the assessment of osteoporosis: expert agreement on current status. The International Quantitative Ultrasound Consensus Group. Journal of bone and mineral research : the official journal of the American Society for Bone and Mineral 
Research. 1997;12(8):1280-8.

30. Lee HD, Hwang HF, Lin MR. Use of quantitative ultrasound for identifying low bone density in older people. Journal of ultrasound in medicine : official journal of the American Institute of Ultrasound in Medicine. 2010;29(7):1083-92.

31. Hosokawa A, Otani T. Ultrasonic wave propagation in bovine cancellous bone. The Journal of the Acoustical Society of America. 1997;101(1):558-62.

\section{Abbreviations}

CAS: computer assisted surgery; SNRs: signal-to-noise ratios; BMD: Bone mineral density; A-mode: Amplitude Mode; SMI: Structural model index; Tb. Th: Trabecular thickness; Th. N: Trabecular number; Th. Sp: Trabecular separation; BV/TV: Bone volume/tissue volume; RF: radio frequency; SD: standard deviation; LSD: least significant difference.

\section{Declarations}

\section{Ethics approval and consent to participate}

This work was approved by the ethics committees of Suzhou Municipal Hospital.

\section{Consent for publication}

All the authors of the paper approved the publication of the article.

\section{Availability of data and materials}

Data related to the current study are available from the corresponding author on reasonable request.

\section{Competing interests}

The authors declare that they have no competing interests.

\section{Funding}

This work was supported by the Key Project of Social Development of Jiangsu province of China -- Clinical Frontier Technology (Grant No. BE2017661), the 333 
Talents Project of Jiangsu province of China (Grant No. BRA2017057). The funding body played no role in the design of the study and collection, analysis, and interpretation of data and in writing the manuscript.

\section{Authors' contributions}

SL and PL conducted all experiments, integrated data, edited figures, and wrote the manuscript; WS helped with the data collection. YJ, ZL and WL contributed a lot to the revision of the manuscript. YC and JF provided essential assistance; JS directed this study, designed the research and gave key advices. The authors read and approved the final manuscript.

\section{Acknowledgements}

None.

\section{Author details}

1. Department of Orthopedic Surgery, the Affiliated Suzhou Hospital of Nanjing Medical University; Suzhou Municipal Hospital. Suzhou, Jiangsu, 215002, People's Republic of China.

2. Academy for Engineering \& Technology, Fudan University. Shanghai, China

3. Suzhou Institute of Biomedical Engineering and Technology (SIBET), Chinese Academy of Science. Suzhou, Jiangsu, 215163, People's Republic of China.

4. Department of Orthopedic Surgery, Suzhou Ninth People's Hospital. Suzhou, Jiangsu, 215000, People's Republic of China.

\section{Figure Legends}

Fig. 1 Micro-CT and physical image of bone block. (A) Micro-CT image of human allograft bone. (B) Physical image of human allograft bone.

Fig. 2 The relationship between bone thickness and penetration amplitude at different frequencies: (A) 2.2MHz; (B) 2.5MHz; (C) 3MHz; (D) 12MHz. $\mathrm{n}=3$. 
569 Fig. 3 The relationship between bone thickness and acoustic attenuation at different 570 frequencies: (A) 2.2MHz; (B) $2.5 \mathrm{MHz}$; (C) $3 \mathrm{MHz}$; (D) $12 \mathrm{MHz} . \mathrm{n}=3$.

571 Fig. 4 Variation of sound attenuation coefficients at different frequencies in the same 572 bone sample: (A) Bovine bone 1; (B) Bovine bone 2; (C) Human allograft bone(high 573 density); (D) Human allograft bone(low density). n=3.

574 Fig. 5 The sound velocity of each bone block under different ultrasonic frequencies:

575 (A) Bovine bone 1 and bovine bone 2; (B) Human allograft bone (high density) and 576 human allograft bone (low density). $n=3$.

577 Fig. 6 The velocity of sound varies with different thickness of the same bone sample: 578 (A) $2.2 \mathrm{MHz}$; (B) $2.5 \mathrm{MHz}$; (C) $3 \mathrm{MHz}$; (D) $12 \mathrm{MHz} . \mathrm{n}=3$.

579 Fig. 7 Experimental Systems. (A) Physical image of the experimental system. (B) 580 Schematic image of the experimental system.

581 Fig. 8 The standard substitution technique: C-In vitro tissue samples; T1、T2-Transmit 582 and receive transducers, respectively; G-Holder; B1-Constant temperature water bath; 583 B2-Degassed distilled water container.

584 Fig. 9 Typical ultrasound backscattered signals received from the ultrasonic target.

585 Fig. 10 Reference signal and transmission signal at different ultrasonic frequencies. 
Figures
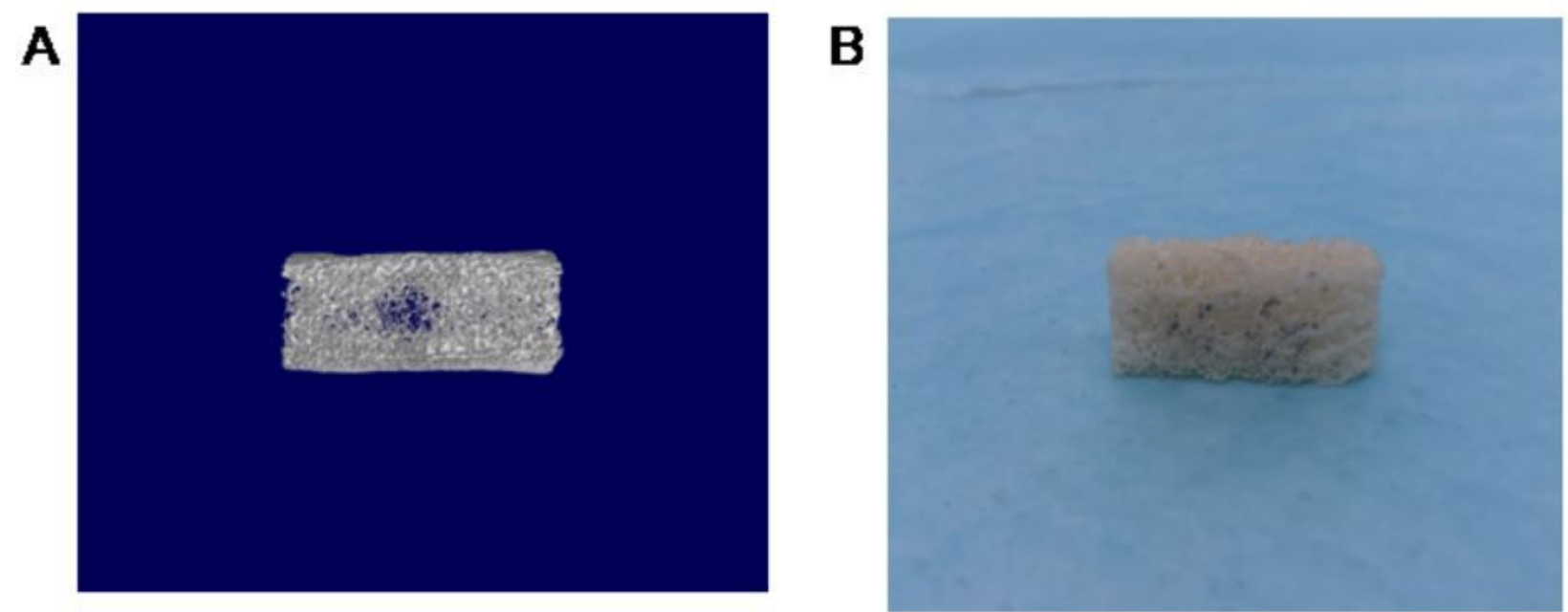

Figure 1

Micro-CT and physical image of bone block. (A) Micro-CT image of human allograft bone. (B) Physical image of human allograft bone. 
A
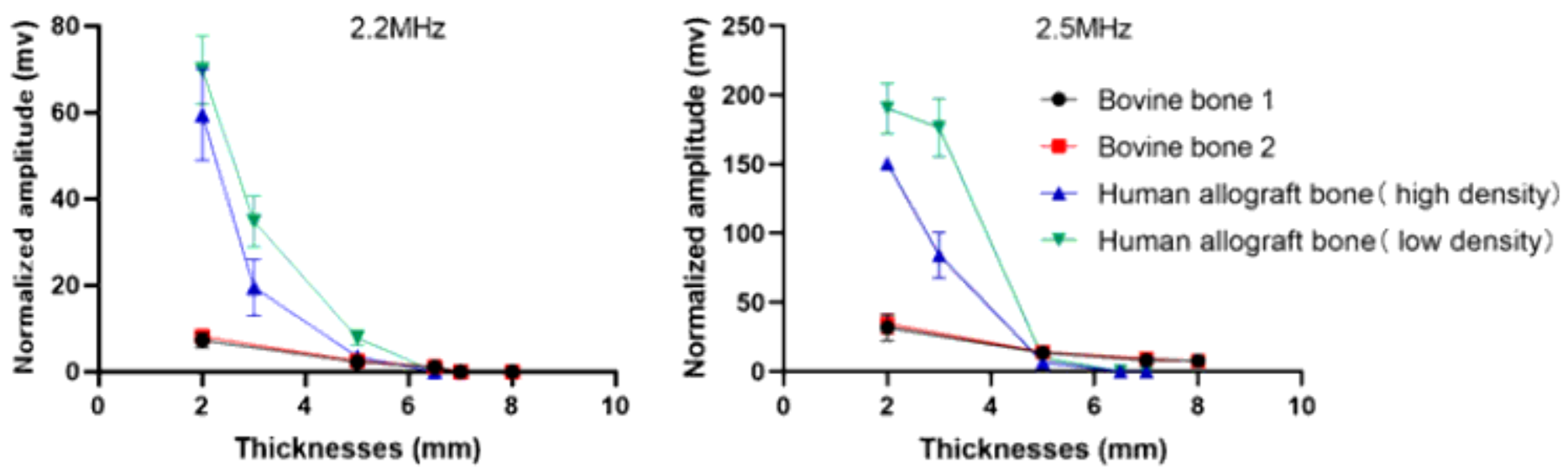

C
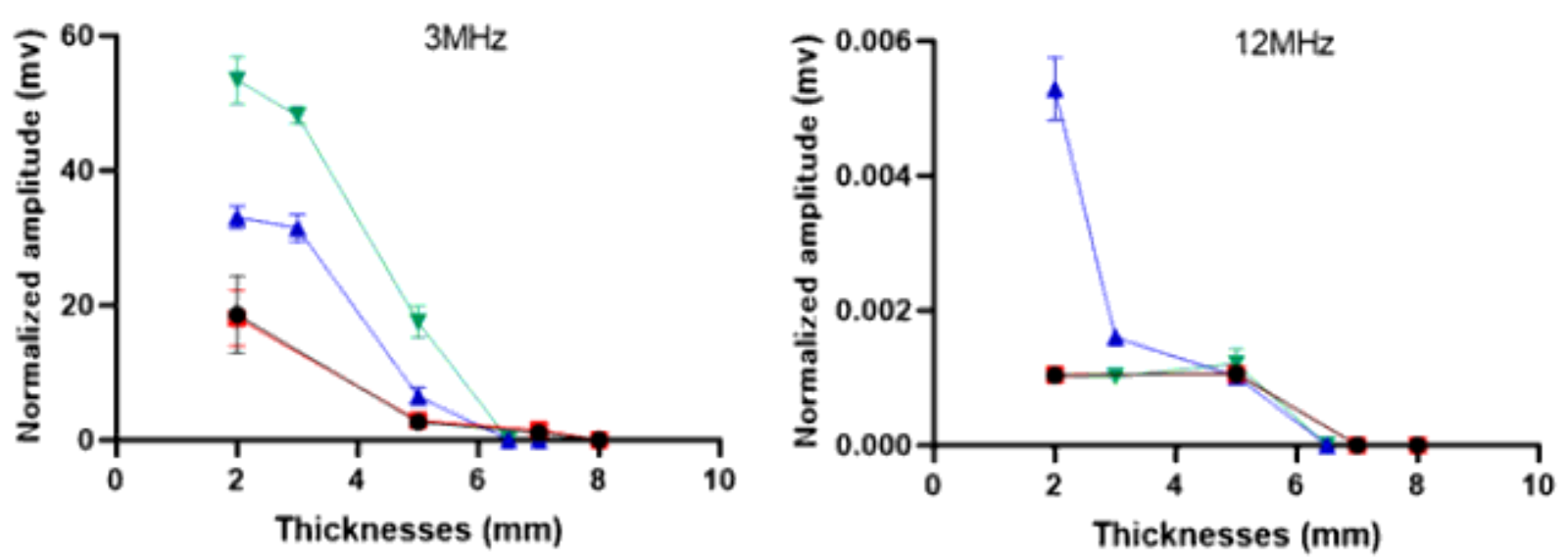

Figure 2

The relationship between bone thickness and penetration amplitude at different frequencies: $(A) 2.2 \mathrm{MHz}$; (B) $2.5 \mathrm{MHz}$; (C) $3 \mathrm{MHz}$; (D) $12 \mathrm{MHz} . \mathrm{n}=3$. 
A

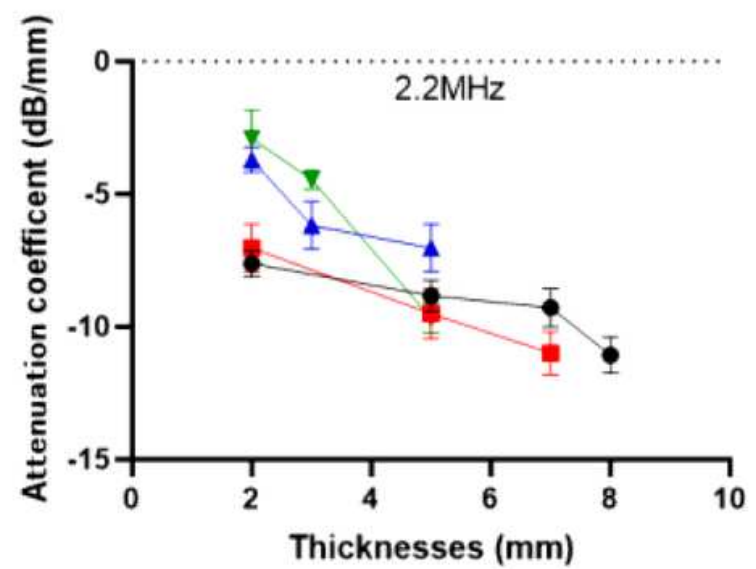

C

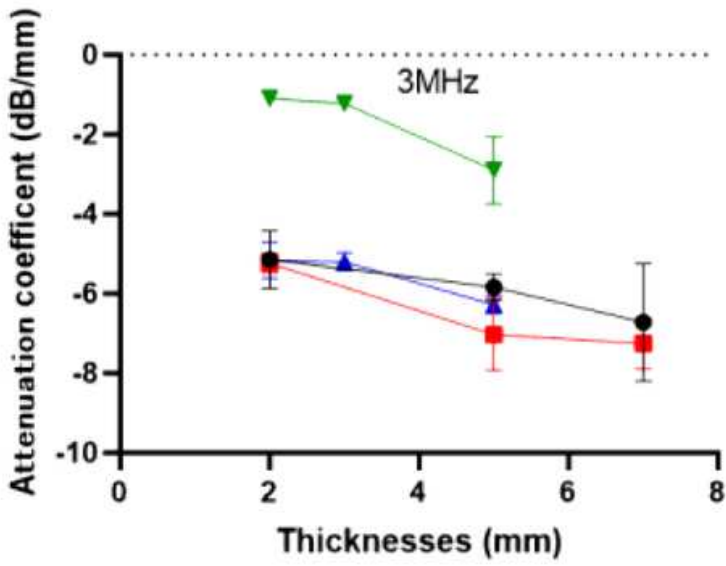

B
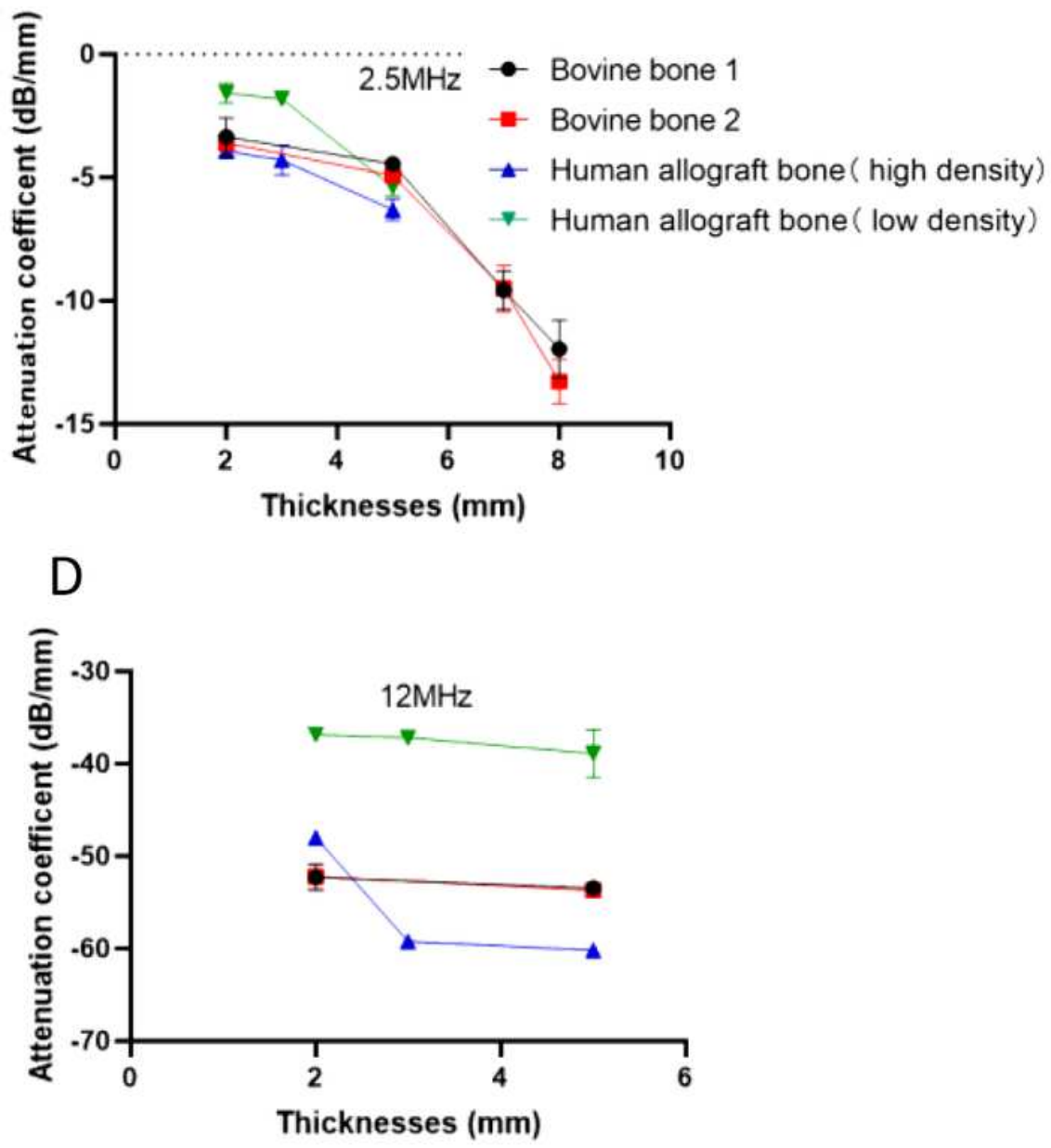

Figure 3

The relationship between bone thickness and acoustic attenuation at different frequencies: (A) $2.2 \mathrm{MHz}$; (B) $2.5 \mathrm{MHz}$; (C) $3 \mathrm{MHz}$; (D) $12 \mathrm{MHz} . \mathrm{n}=3$. 
A

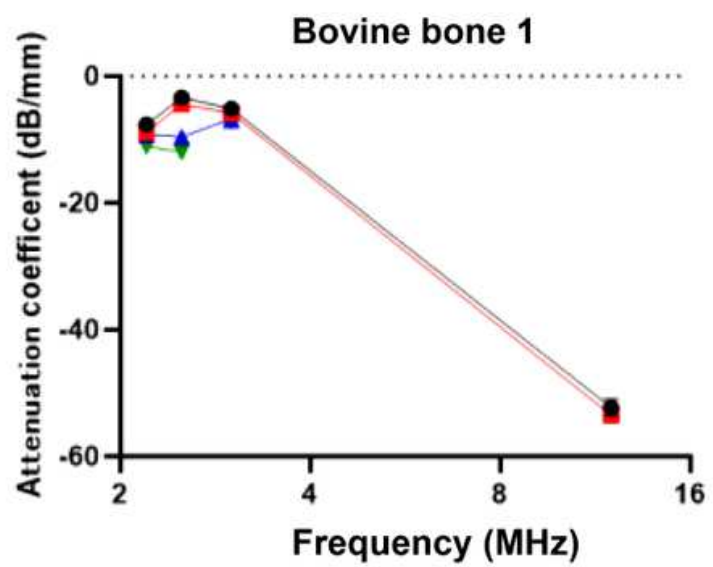

C

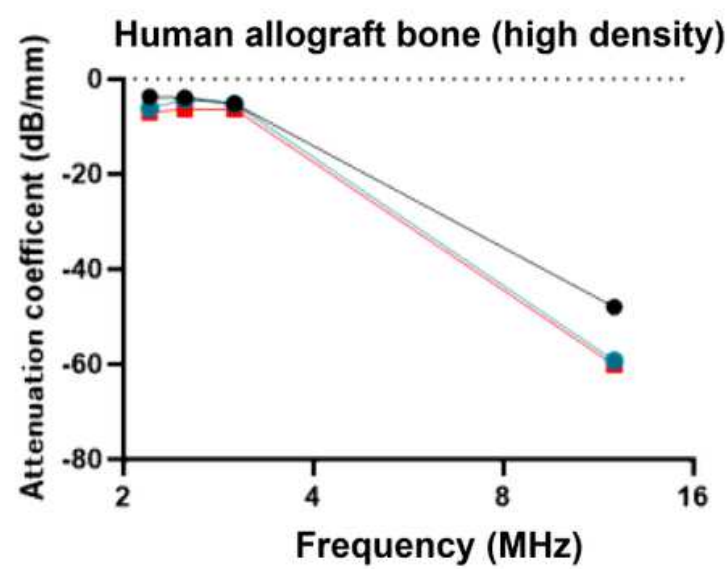

$\mathrm{B}$

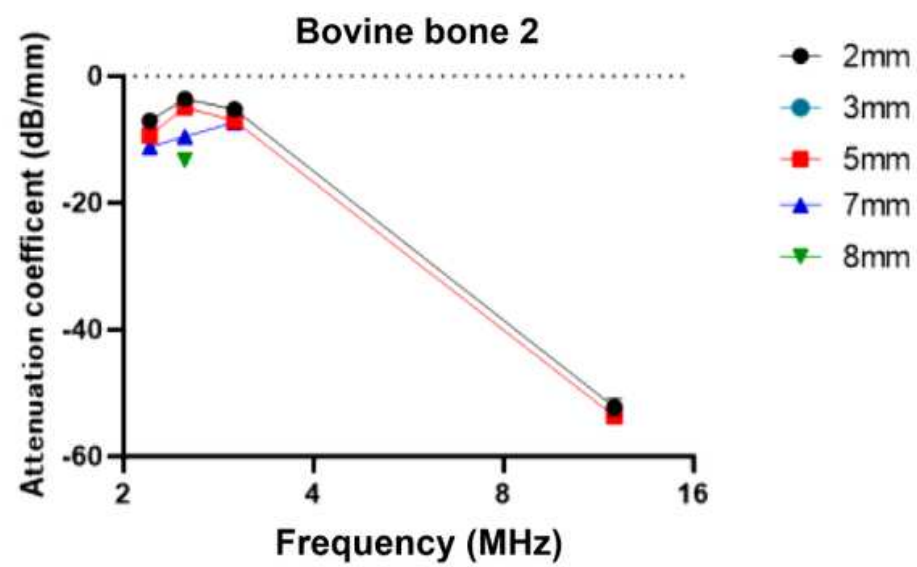

D

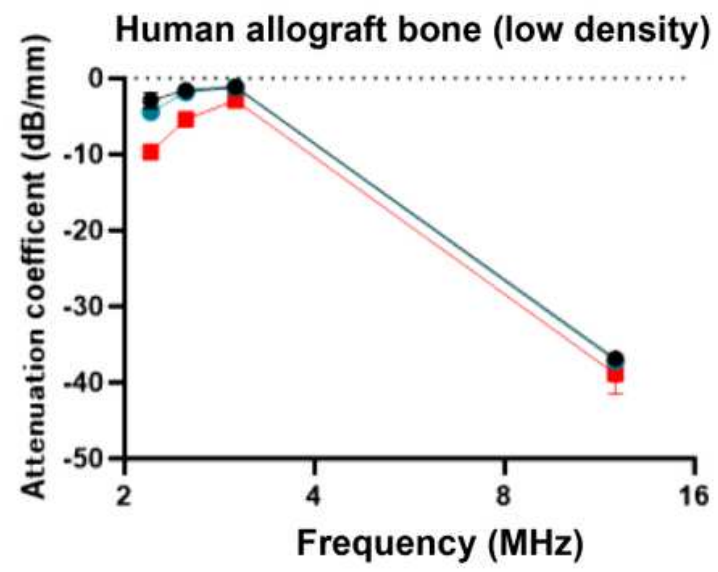

Figure 4

Variation of sound attenuation coefficients at different frequencies in the same bone sample: $(A)$ Bovine bone 1; (B) Bovine bone 2; (C) Human allograft bone(high density); (D) Human allograft bone(low density). $n=3$.
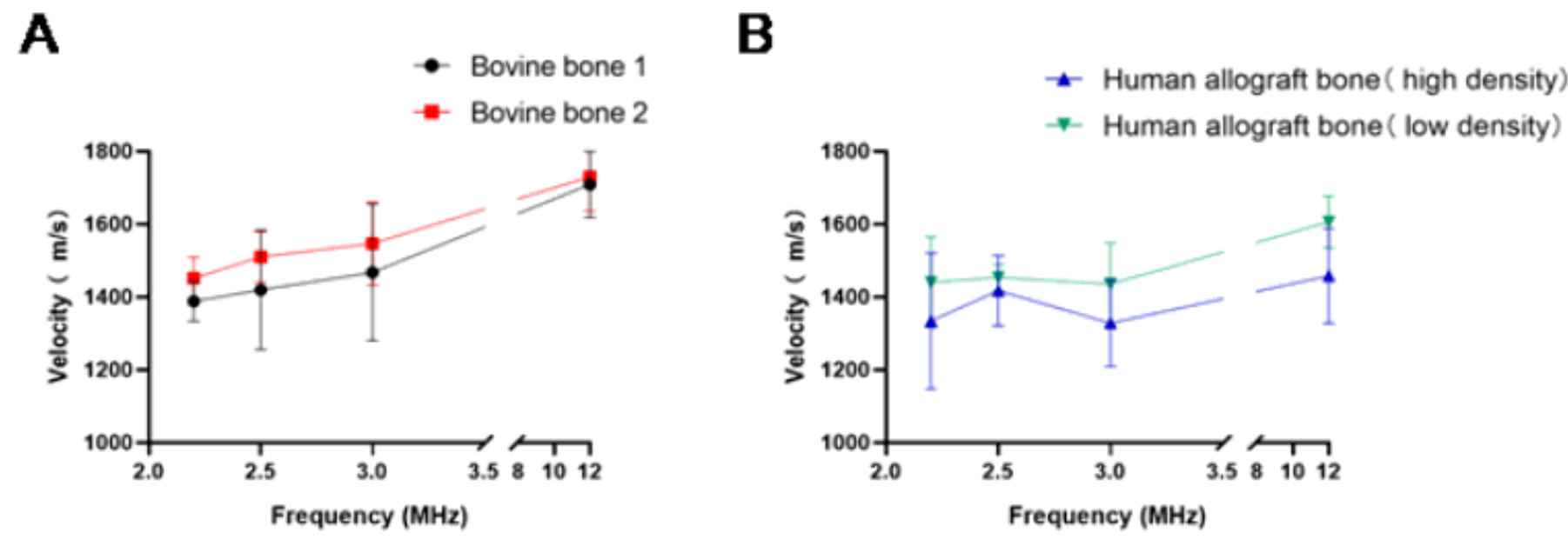
Figure 5

The sound velocity of each bone block under different ultrasonic frequencies: $(A)$ Bovine bone 1 and bovine bone 2; (B) Human allograft bone (high density) and human allograft bone (low density). $\mathrm{n}=3$.

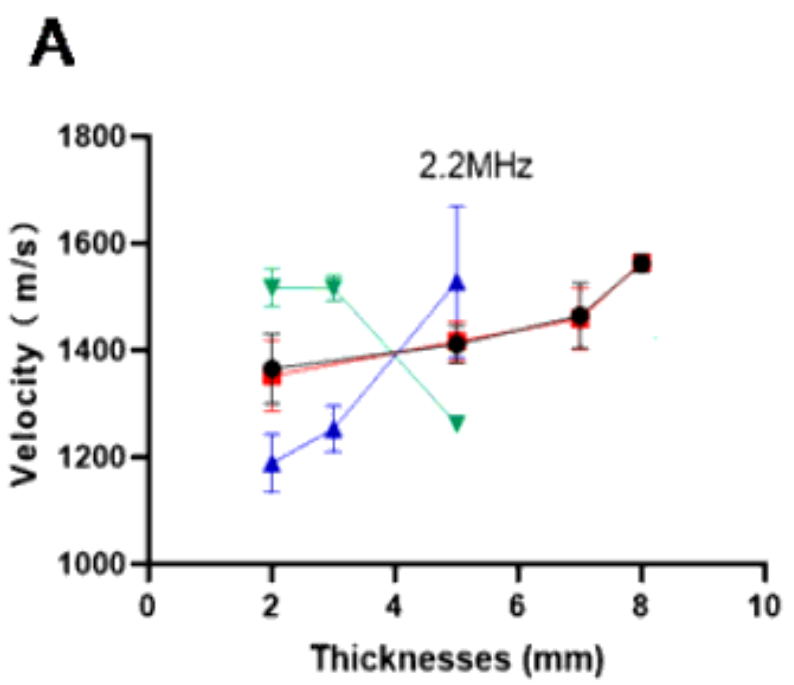

B

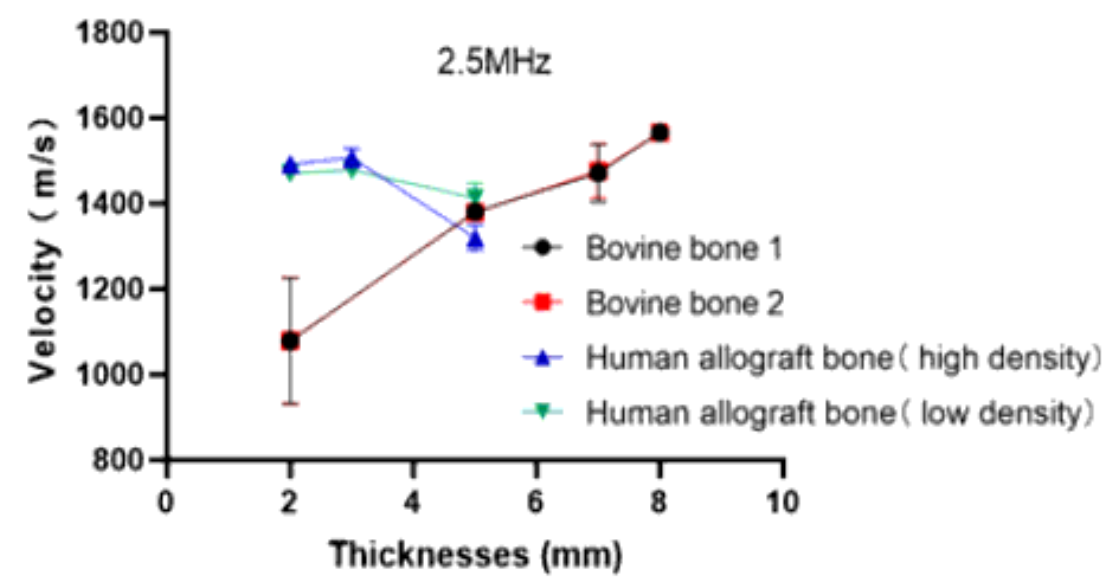

C

D
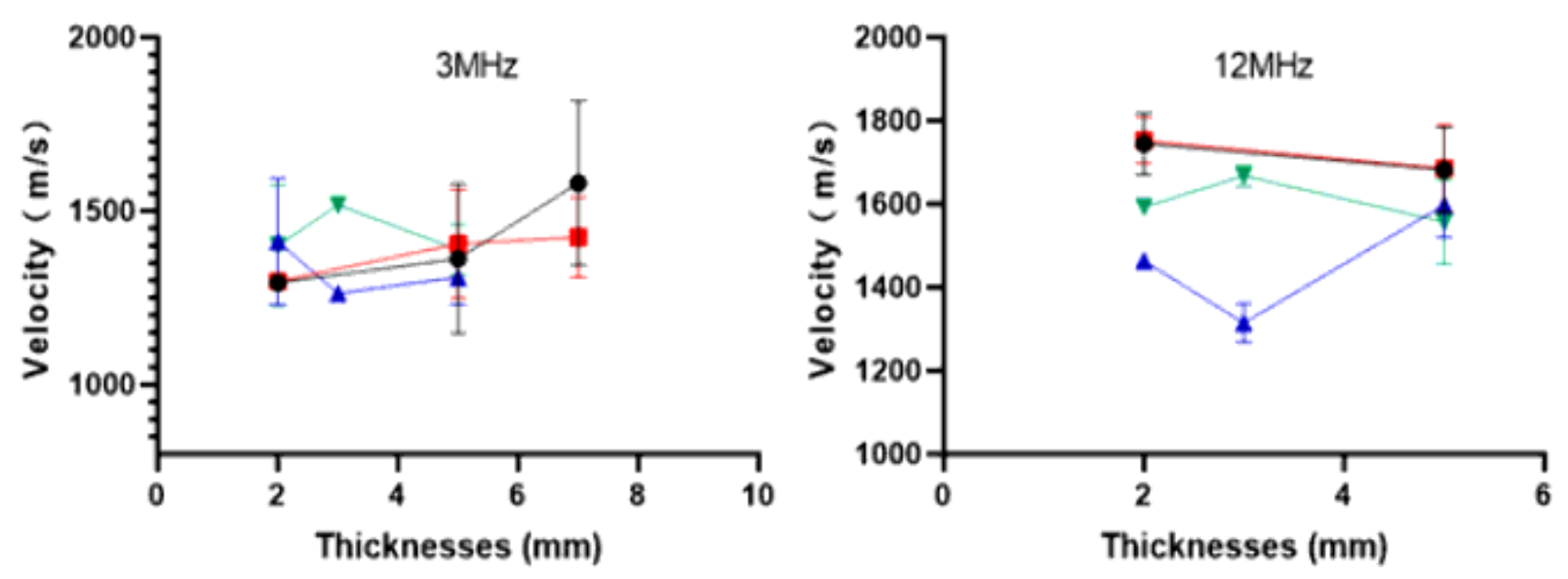

Figure 6

The velocity of sound varies with different thickness of the same bone sample: (A) $2.2 \mathrm{MHz}$; (B) $2.5 \mathrm{MHz}$; (C) $3 \mathrm{MHz}$; (D) $12 \mathrm{MHz} . \mathrm{n}=3$. 

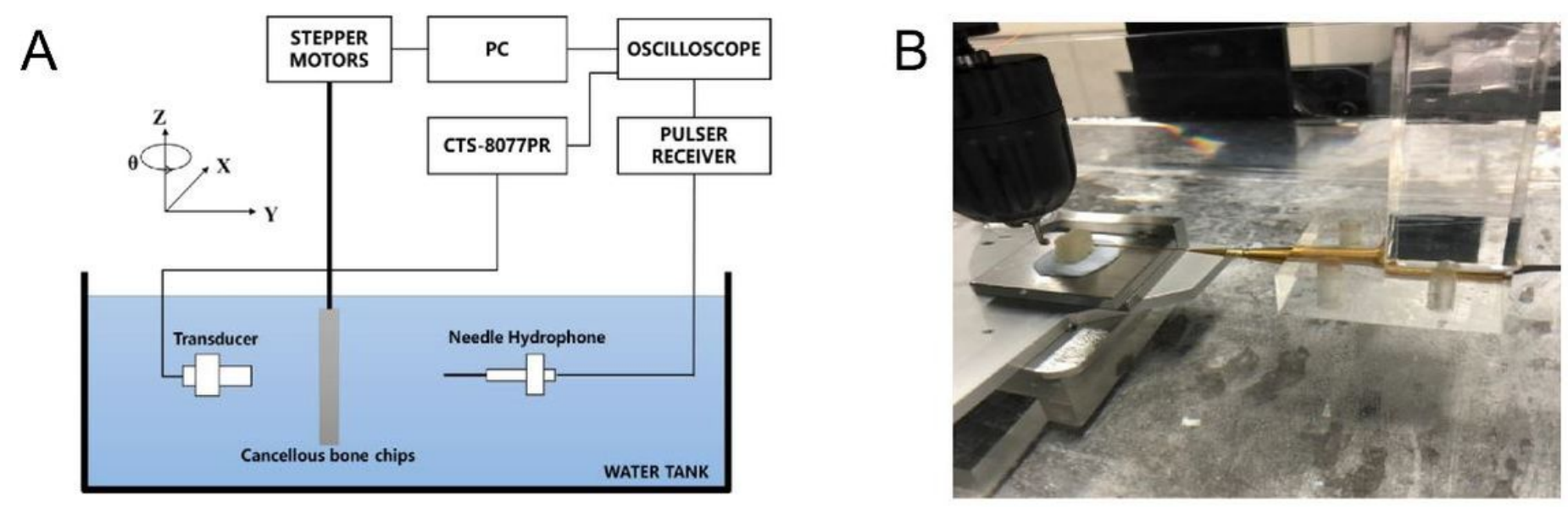

Figure 7

Experimental Systems. (A) Physical image of the experimental system. (B) Schematic image of the experimental system. 


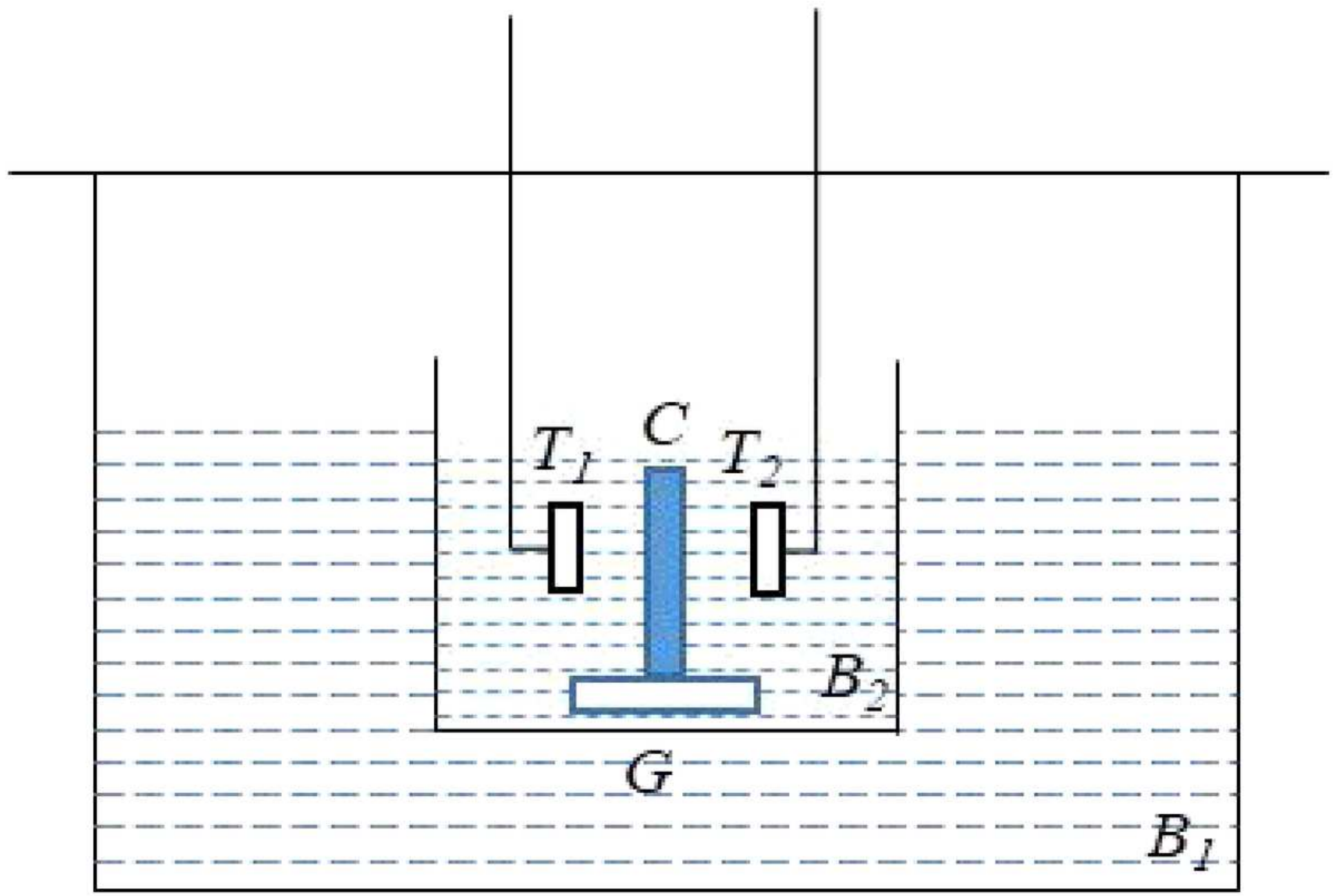

Figure 8

The standard substitution technique: $\mathrm{C}$-In vitro tissue samples; T1DT2-Transmit and receive transducers, respectively; G-Holder; B1-Constant temperature water bath; B2-Degassed distilled water container. 

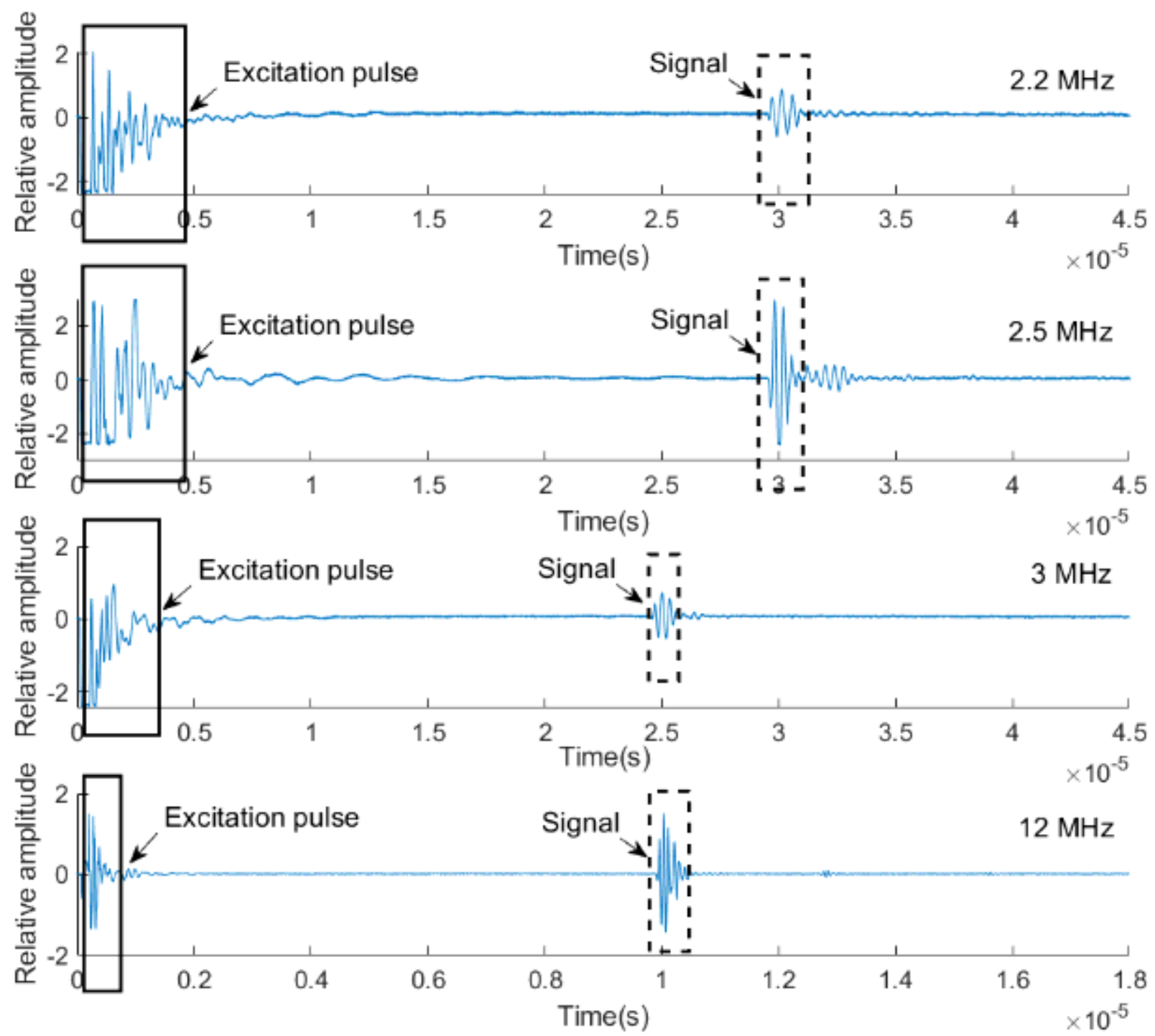

Figure 9

Typical ultrasound backscattered signals received from the ultrasonic target. 

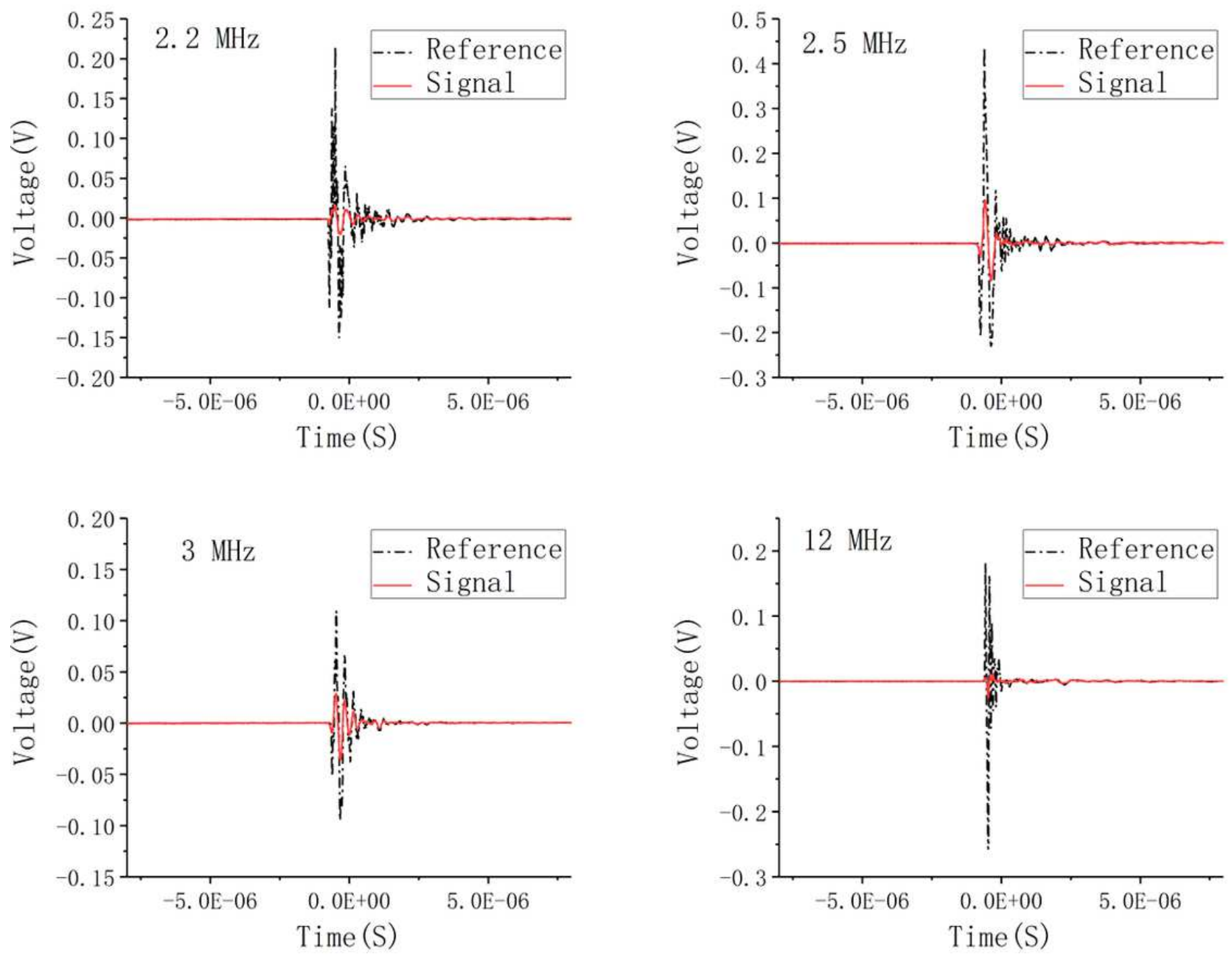

Figure 10

Reference signal and transmission signal at different ultrasonic frequencies. 